\title{
Stress responses to conspecific visual cues of predation risk in zebrafish
}

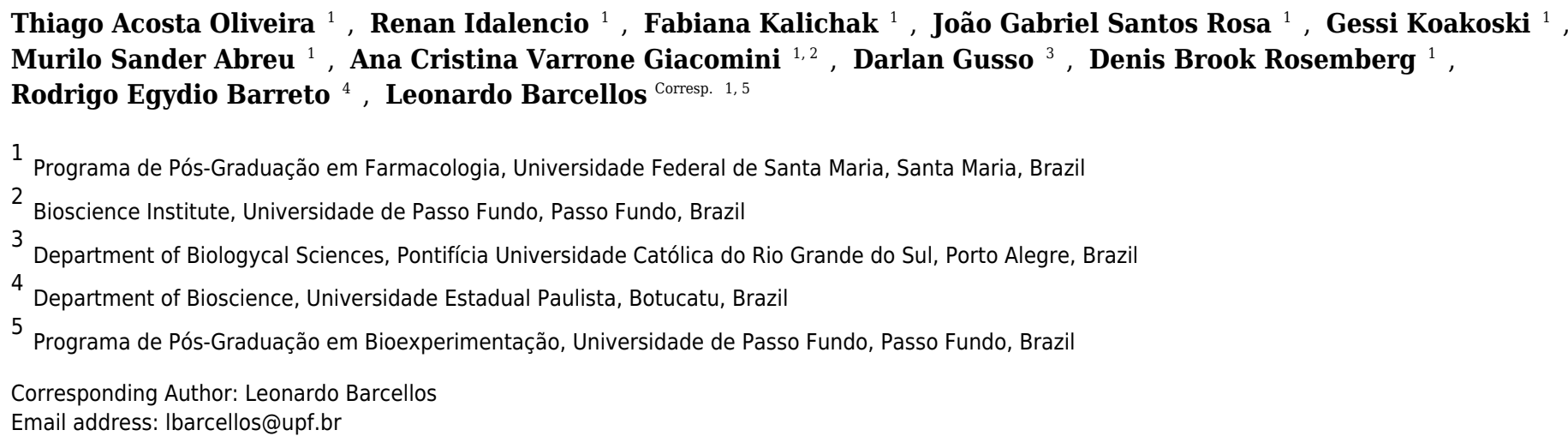

Chemical communication relating to predation risk is a trait common among fish species. Prey fish under threat of predation can signal risk to conspecific fish, which then exhibit defensive responses. Fish also assess predation risk by visual cues and change their behavior accordingly. Here, we explored whether these behavioral changes act as visual alarm signals to conspecific fish that are not initially under risk. We show that shoals of zebrafish (Danio rerio) visually exposed to a predator display antipredator behaviors. In addition, these defensive maneuvers trigger antipredator reactions in conspecifics and, concomitantly, stimulate the hypothalamus-pituitary-interrenal axis, leading to cortisol increase. Thus, we conclude that zebrafish defensive behaviors act as visual alarm cues that induce antipredator and stress response in conspecific fish. 
1 Stress responses to conspecific visual cues of predation risk in

2 zebrafish

3

4

8

9 10

Thiago Acosta Oliveira ${ }^{1}$; Renan Idalencio ${ }^{1}$, Fabiana Kalichack ${ }^{1}$, João Gabriel S. da Rosa ${ }^{1}$, Gessi Koakoski $^{1}$, Murilo S. de Abreu ${ }^{1}$, Ana Cristina V. Giacomini ${ }^{1,2}$, Darlan Gusso ${ }^{3}$, Denis B. Rosemberg ${ }^{1}$, Rodrigo E. Barreto ${ }^{4}$, Leonardo José Gil Barcellos*1,5

1Programa de Pós-Graduação em Farmacologia, Universidade Federal de Santa Maria, Santa Maria, Brazil

2Bioscience Institute, Universidade de Passo Fundo, Passo Fundo, Brazil

3Department of Biological Sciences, Pontifícia Universidade Católica do Rio Grande do Sul, Porto Alegre, Brazil

4 Department of Bioscience, Universidade Estadual Paulista, Botucatu, Brazil

5Programa de Pós-Graduação em Bioexperimentação, Universidade de Passo Fundo, Passo Fundo, Brazil

* Correspondence to:

Dr. Leonardo J.G. Barcellos

e-mail address: 1barcellos@upf.br

Programa de Pós-Graduação em Bioexperimentação, Universidade de Passo Fundo (UPF), BR 285, São José, Passo Fundo, RS, Brazil, 99052-900

Tel: +55543316.8100

Fax: +55 543316.848 


\section{Abstract}

45 Chemical communication relating to predation risk is a trait common among fish species. Prey fish 46 under threat of predation can signal risk to conspecific fish, which then exhibit defensive 47 responses. Fish also assess predation risk by visual cues and change their behavior accordingly.

48 Here, we explored whether these behavioral changes act as visual alarm signals to conspecific fish 49 that are not initially under risk. We show that shoals of zebrafish (Danio rerio) visually exposed 50 to a predator display antipredator behaviors. In addition, these defensive maneuvers trigger 51 antipredator reactions in conspecifics and, concomitantly, stimulate the hypothalamus-pituitary52 interrenal axis, leading to cortisol increase. Thus, we conclude that zebrafish defensive behaviors 53 act as visual alarm cues that induce antipredator and stress response in conspecific fish. 
56

57

\section{Introduction}

Prey-predator interactions occur throughout the animal kingdom (Cresswel, 2010), with every interaction involving both unique and general characteristics. Such interactions occur in multiple directions (prey to predator, predator to prey, prey to prey, and even predator to predator) (Barcellos et al., 2014, Mullan et al., 2015, Dunlop-Hayden \& Rehage, 2011). Prey can access predators through a diversity of signals that can be visual, olfactory, acoustical, and vibratory (Barcellos et al., 2014, Barreto et al., 2003). There is a vast amount of information in the scientific literature about these types of perceptions in fish (Wisendem et al., 2004, Barcellos et al., 2011) in response to the presence of a predator, and in conspecific fish threatened by a predator (Jordão, 2000). Different combinations of these signals in the presence of a predator, or even the diverse forms of communication about a predator threat, between conspecific prey induce different antipredator maneuvers (O`Connor et al., 2015).

During prey-predator interactions, early detection of predation risk is crucial for prey survival (Allen, 1975), and chemical communication plays a key role in such risk assessments (Chivers \& Smith, 1998). Prey fish display antipredator behaviors when they perceive the odor of a predator (direct perception; e.g., Miyai et al., 2016) or when alerted to the presence of a predator via chemical cues released by other individuals (indirect perception; e.g., Barcellos et al., 2011 and 2014; Barreto et al., 2013; Oliveira et al., 2014). Although an enormous amount of research has been undertaken on chemical communication among prey, many questions remain unanswered. In addition to chemical cues, prey fish also detect predators via visual cues and display appropriate defensive reactions in response to these threats (Kalluef et al., 2014), but these responses are far less explored in the literature. 

et al., 2003; Gerlai, 2003; Barcellos et al., 2007; Miller \& Gerlai, 2007; Gebauer et al., 2011). As a diurnal fish species, zebrafish (Danio rerio) clearly use this sensorial modality for communication with conspecifics. Eavesdropping is one type of visual communication, wherein individuals use the available visual information provided by conspecific fish behaviors (Abril-deAbreu et al., 2017) to evaluate the context and appropriate response behavior. It has been previously shown that eavesdropping influences zebrafish behavior (Abril-de-Abreu et al., 2017). In this context, we propose that predator-induced behavioral changes could act as visual alarm signals that provoke defensive reactions in conspecific fish. Here, we show that antipredator behaviors displayed by zebrafish are visual alarm cues that induce defensive maneuvers and surges in the production of cortisol in unthreatened conspecifics.

\section{Materials and Methods}

\subsection{Ethical note}

This study was approved (protocol \#20/2016) by the Ethics Commission for Animal Use of the Universidade de Passo Fundo (Passo Fundo, RS, Brazil), and all methods were carried out in accordance with the guidelines of National Council of Animal Experimentation Control (CONCEA).

\subsection{Zebrafish and housing conditions}

Wild-type zebrafish adults ( \pm 8 months) of both sexes, averaging $\pm 5 \mathrm{~cm}$ in length and \pm $0.4 \mathrm{~g}$ in weight, were maintained under a photoperiod of $\sim 14 \mathrm{~h}$ light/10 $\mathrm{h}$ dark in indoor holding $\operatorname{tanks}\left(2\right.$ fish/L). Water conditions were maintained as follows: temperature: $28.0 \pm 2.0{ }^{\circ} \mathrm{C} ; \mathrm{pH}: 7.0$ \pm 0.6 ; dissolved oxygen: $6.8 \pm 0.4 \mathrm{mg} / \mathrm{L}$; total ammonia: < $0.01 \mathrm{mg} / \mathrm{L}$; total hardness: $6 \mathrm{mg} / \mathrm{L}$; and 
101 alkalinity: $22 \mathrm{mg} / \mathrm{L}$ of $\mathrm{CaCO}_{3}$. The fish were fed twice daily (09:00 h and 16:00 h) with commercial

102 flakes (TetraMin ${ }^{\circledR}$, Tetra, Melle, Germany) until satiation. The dimensions of the aquaria were 13

$103 \mathrm{~cm}$ length $\times 30 \mathrm{~cm}$ width $\times 40 \mathrm{~cm}$ height, and contained a total water volume of $13 \mathrm{~L}$; stock density

104 was similar to the holding tanks ( 2 fish/L). The experimental chambers measured $40 \mathrm{~cm}$ length $\times$

$10530 \mathrm{~cm}$ width $\times 30 \mathrm{~cm}$ height, and contained $30 \mathrm{~L}$ of water; stock density was $1 \mathrm{fish} / 3 \mathrm{~L}$.

106 Each experiment was performed with four replicates and consisted of six groups containing 10710 fish each (for a total of 240 fish). Additional controls were performed in triplicate, using two 108 groups of 10 fish each (for a total of 60 fish). Thus, 300 fish were used in the experiments. For 109 cortisol measurements, two data points were generated from each replicate (2-3 fish per data point) 110 and then pooled into a single group, which was used for the statistical analysis.

\section{1}

112

\subsection{Experimental design and procedures}

Zebrafish shoals were maintained either in a chamber that allowed visualization of a predator/non-predator fish or in a tank devoid of other fish. We called fish kept under these conditions "sender fish" (SF, Fig. 1). In an adjacent chamber, we placed other zebrafish shoals were placed in an adjacent chamber that only permitted visualization of the SF shoal. Fish kept under these conditions were termed "receiver fish" (RF, Fig. 1).

The chambers were tightly sealed to avoid potential chemical communication between fish in different chambers. Three experimental conditions were thus established: in the first, RF were evaluated during SF visual exposure to a predatory fish (the tiger oscar Astronotus ocellatus); in the second, a harmless fish (the goldfish Carassius auratus) was used to test whether visualization of any fish provoked behavioral changes in SF and, consequently, in RF. Lastly, SF were exposed to the view of a water-filled aquarium devoid of fish, which served as the control. 

view the predator directly. Two groups of RF were isolated (without the visual cues emanating remained in its chamber 1 , and a second group of RF was kept in their chamber 3 while the nonpredator fish (goldfish) remained in its respective chamber 1 . Sample size was $n=10$ for each condition. Initially, SF and RF zebrafish shoals and stimulus fish (non-predator and predator) were introduced into their respective chambers for an adaptation period of $24 \mathrm{~h}$, during which SF fish could not see the stimulus chamber (predator fish, harmless fish, or tank with only water), whereas

RF were allowed to view SF during the entirety of the adaptation and experimental periods. Visual contact was blocked using an opaque plastic plaque. Following the adaptation period, the plaque was removed, thereby allowing visualization between the stimulus chamber and SF for $60 \mathrm{~min}$, which was recorded on video for later behavioral analysis. After the visualization period, both SF and RF fish were captured, killed by immediate spinal section, frozen in liquid nitrogen, and stored at $-20{ }^{\circ} \mathrm{C}$ until required for use in whole-body cortisol assays. was not changed, and the fish were not fed during the experiment itself to avoid the effects of handling procedures, as fish remained in the chambers for only $24 \mathrm{~h}$. No exchange of water occurred between the chambers; prior to the experimental trials, the chambers were filled with water and it was observed that adjacent chambers remained completely dry, thus ensuring that communication between fish within each chamber occurred solely via visualization. 


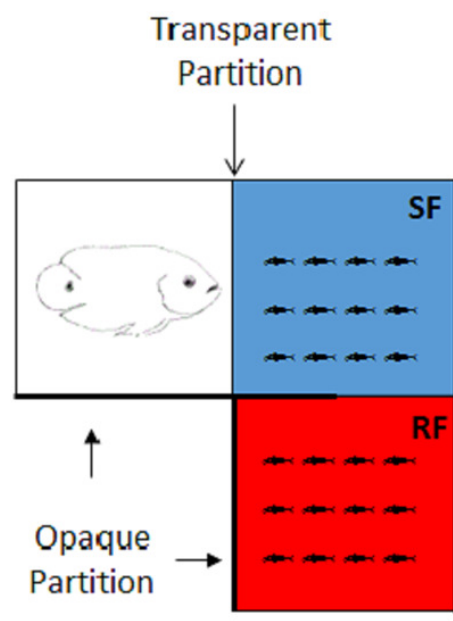

SF with visual contact

with a predator

RF with visual contact

with SF with visual

contact with a predator

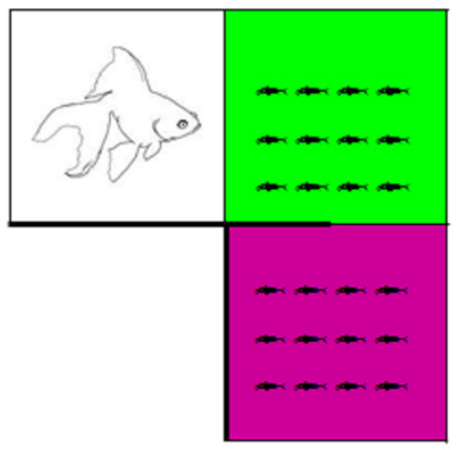

SF with visual contact

with a non-predator

$\mathrm{RF}$ with visual contact

with SF with visual contact

with a non-predator

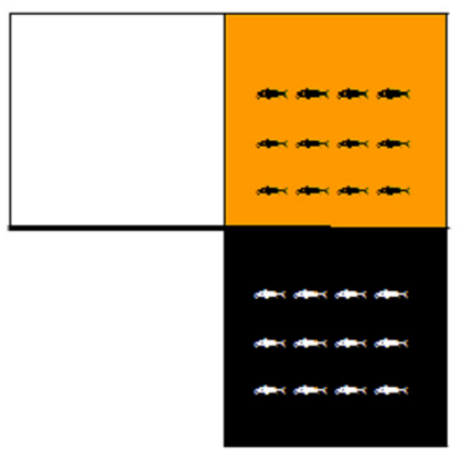

SF control fish

RF control fish

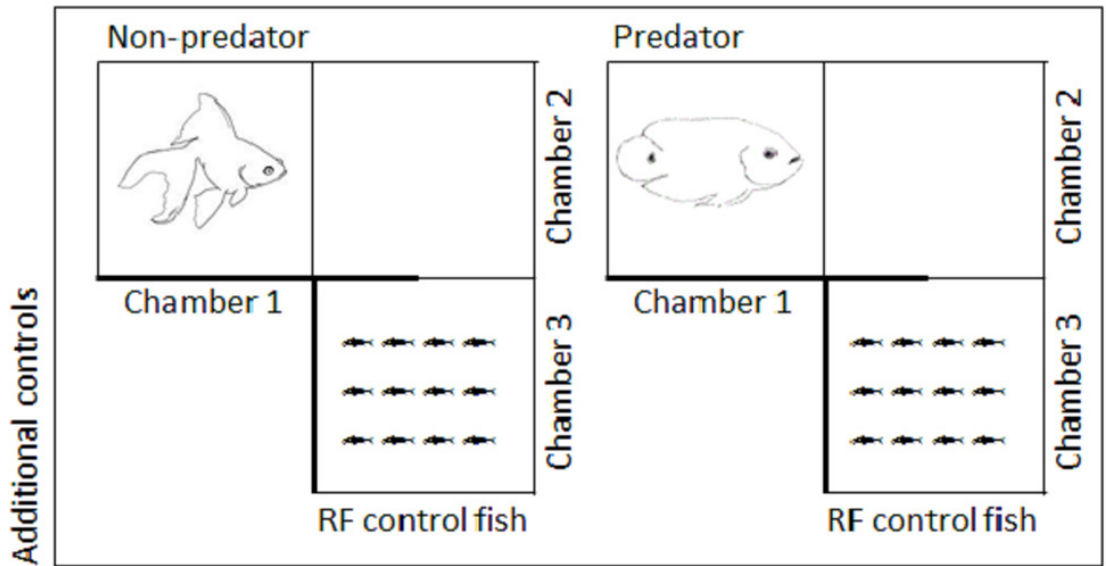

Predator-Tiger Oscar (Astronotus ocellatus)

- Sender (SF) or receiver (RF) zebrafish

Non Predator - Goldfish (Carasssius auratus) 
145 Figure 1. Schematic representation of the experimental conditions. Fish illustrations were drawn 146 by LB.

147

\subsection{Cortisol extraction and determination}

Whole-body cortisol levels were determined in 4-6 fish specimens taken from each experimental group of each replicate, for a total of 16-24 fish. Cortisol sample sizes varied because whole-body cortisol analysis requires a minimum of $0.5 \mathrm{~g}$ of tissue extract; given the small size of these zebrafish, we used pooled samples of 2-3 fish, with the goal of attaining a final sample size of eight.

Tissue cortisol levels were used as an indicator of stress response. To minimize potential handling-induced stress responses, the period between capture and killing was $<30 \mathrm{~s}$. Each killed fish was weighed, minced, and placed in a tube containing $3 \mathrm{ml}$ of phosphate buffered saline (PBSg, $\mathrm{pH}$ 7.3). The contents were then transferred to a tube containing ethyl ether and subjected to vortexing, then centrifuged after immediately being frozen in liquid nitrogen. The thawed portion (cortisol containing diethyl ether) was decanted and transferred to a new tube and allowed to evaporate completely to yield a lipid extract containing cortisol, which was then stored at -20 ${ }^{\circ} \mathrm{C}$.

Tissue extracts were re-suspended in PBSg and whole-body cortisol levels were measured using an ELISA kit (EIAgen TM cortisol test, BioChemImmunosystems). This kit was previously shown to be effective for analysis of zebrafish tissue extracts by Sink et al. (2007). Accuracy was tested by calculating cortisol recovery in samples spiked with known amounts of cortisol; precision was assessed by calculating the intra-assay coefficient of variation (CV) of 12 repeated assays in 
167 seven randomly chosen samples on the same plate; and reproducibility was evaluated by assaying 168 the same samples on different plates and calculating the inter-assay CV.

To test for linearity and parallelism, serial dilutions of tissue extracts were performed in

170

171

172

173

174

175

176

177

178

179

180

181

182

183

184

185

186

187

188

189

\subsection{Behavioral quantification}

We used the methodology described by Oliveira et al. (2014) to quantify the behavioral responses. Briefly, the water column was divided into three sections of equal size from the bottom to the surface. The time that fish spent in the bottom area was observed and manually recorded by a single experienced observer who was unaware of what group was being assessed, with the percentage of the session time for each of these behaviors later calculated. The rationale for quantifying fish behavior via observation and manual recording was based on the findings of Speedie \& Gerlai (2008), which clearly show that zebrafish responses to alarm substances can be reliably quantified by visual-manual recording as well as through computerized video-tracking methods. We characterized time spent near the tank bottom as an indicator of defensive reactions (Gerlai \& Csányi, 1990; Gerlai et al., 2000; Quadros et al., 2016), with the duration of this behavior expressed as a percentage of the total observation session duration. Onset of the time at the tank bottom was considered to occur when at least three of the 10 zebrafish remained within the bottomthird section, in accordance with the methods described by Speedie \& Gerlai (2008).

\subsection{Statistical analysis}


For whole-body cortisol and behavior values, we compared all treatments and also

191

192

193

194

195

196

197

198

199

200

201

202

203

204

205

206

207

208

209

210

211

212

performed comparisons between SF zebrafish senders and between RF zebrafish under experimental conditions. Regarding cortisol values and time spent in the bottom section of the aquaria, we applied a two-way ANOVA, with type of stimulus fish and category (SF or RF) of zebrafish as independent factors, followed by Tukey's multiple comparison test to compare the means in each experiment. Significant differences were set at $\mathrm{P}=0.05$. To compare the $\mathrm{SF}$ and $\mathrm{RF}$ zebrafish according to the type of stimulus fish, we applied a one-way ANOVA, followed by Tukey's multiple comparison test or a Kruskal-Wallis test followed by Dunn's multiple comparison test, depending on if data passed the Kolmogorov-Smirnov and Bartlett tests. Significant differences were set at $\mathrm{P}=0.05$.

\section{Results}

We detected a strong interaction between the type of stimulus fish and the category (SF or $\mathrm{RF})$ of zebrafish $\left(\mathrm{P}=0.0077 ; \mathrm{F}_{2,39}=5.527\right)$. We found that visual perception of the predator increased whole-body cortisol in both SF and RF zebrafish compared to the SF control $(\mathrm{P}=0.0077$; SF comparison $\mathrm{P}=0.0006, \mathrm{~K}=14.92$; $\mathrm{RF}$ comparison $\mathrm{P}=0.001, \mathrm{~K}=13.74$ ). This effect did not occur for SF and RF in the non-predator fish treatment, in which increased cortisol was observed only in SF relative to the SF control. The tank devoid of fish (filled with only water) elicited no significant cortisol response in either SF or RF (Figure 2A).

Only the type of stimulus fish had a significant effect on defensive behavior $(\mathrm{P}<0.0001$; $\mathrm{F}_{2,18}=96.09$ ); both SF and RF fish exposed to a predator fish spent more in the bottom section of the tank, whereas SF and RF fish exposed to a non-predator fish also spent more time in the bottom section but this response was less intense (statistically lower) than those SF and RF observed in 
213 predator exposure treatment, considering the comparison of both groups (non-predator and 214 predator treatment) with the control group (non-stimulus fish). The aquaria with only water 215 induced no significant changes in defensive behaviors ( $\mathrm{SF}$ comparison $\mathrm{P}<0.0001, \mathrm{~F}_{2,9}=50.17$; $\mathrm{RF}$ 216 comparison $\left.\mathrm{P}<0.0001, \mathrm{~F}_{5,18}=46.63\right)$. Zebrafish from the two additional controls had very low 217 cortisol concentrations $(2.88 \pm 0.74$ and $3.63 \pm 0.59 \mathrm{ng} / \mathrm{g}$ tissue $)$, levels typically found in non218 stimulated control fish (Barcellos et al., 2007; 2014).

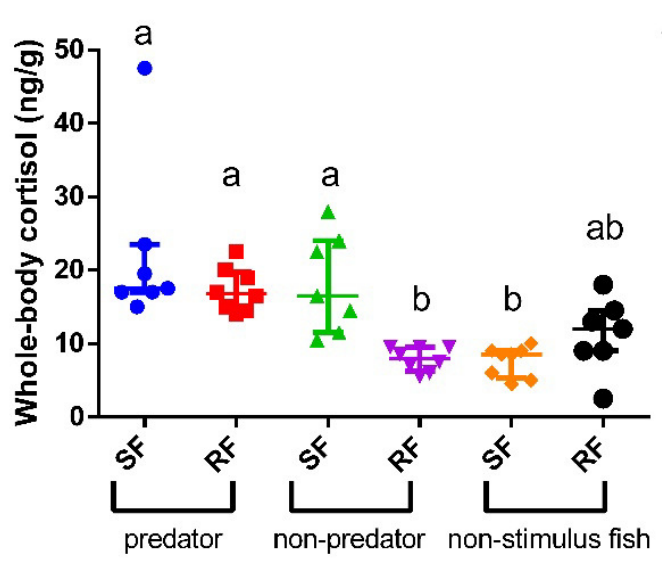

A
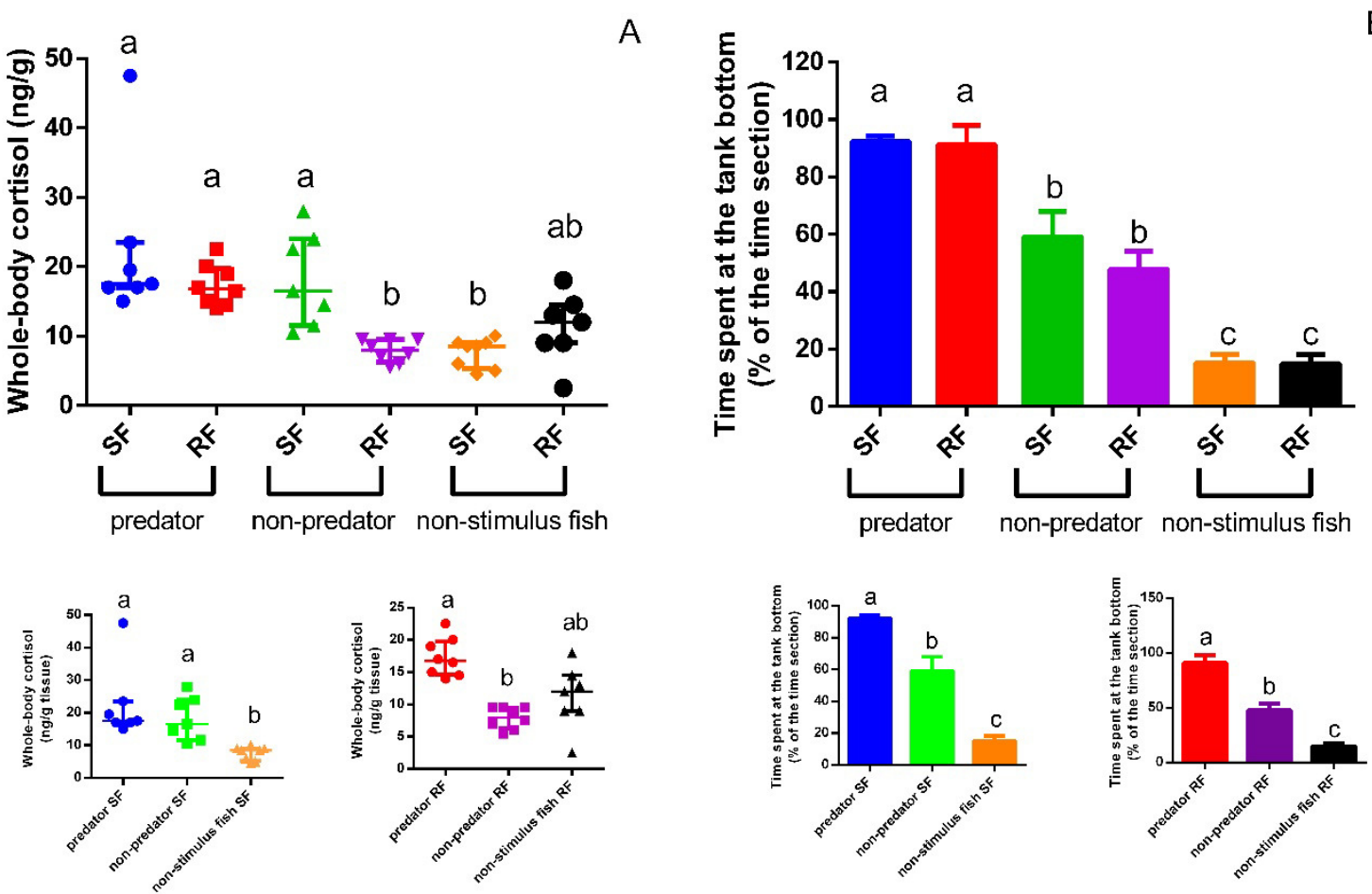

Figure 2. Whole-body cortisol response (median \pm interquartile range) (A) and time spent in the tank bottom (mean \pm SEM) (B) of SF and RF. Data were compared by Kruskal-Wallis complemented by a Dunn's multiple comparisons test (panel A) and by two-way ANOVA

224 followed by Tukey's multiple range test (panel B) ( $\mathrm{n}=7-8$ for cortisol and 4 for behavioral analysis). Different letters above medians or means indicate statistical differences. 
227

228

229

230

231

232

233

234

235

236

237

238

239

240

241

242

243

244

245

246

247

248

\section{Discussion}

Here we demonstrate that zebrafish visually exposed to predators display antipredator behaviors that in turn act as visual alarm cues for conspecifics. Zebrafish unexposed to a predator exhibited defensive maneuvers when allowed to view conspecific individuals displaying defensive behaviors. In addition, these visual cues induced activation of the hypothalamus-pituitaryinterrenal axis (stress response), culminating in increasing levels of whole-body cortisol. These endocrinal and behavioral responses are likely associated with stress events, such as predation risk, as they are well documented by studies of biological interactions between prey and predators in several species (Barton, 2002; Clinchy et al., 2011; Archard et al., 2012). Our results suggest that visual eavesdropping occurs in zebrafish in the context of predation risk. Zebrafish in direct visual contact with a harmless fish, that is, a false predation risk, displayed short-term behavioral alterations and an irreversible stress response. We termed it "irreversible stress response" because we postulated that once triggered, this cortisol response displays a typical increase in cortisol levels in this group of fish, whereas behavioral responses can be quickly adjusted. In contrast, conspecifics observing this altered behavior were able to interpret and process the information as signaling a non-dangerous situation, evidenced by the lack of change in behavioral and endocrine stress responses in RF.

Cortisol levels were elevated in both SF and RF when a predator was visually accessible, presenting a condition of potential imminent predation risk, but cortisol increases were not observed in RF when in visual contact with a non-predator fish or a water-filled tank devoid of fish. Another reliable indicator of anti-predator behavior (Gerlai, 2003) is the time zebrafish spent at the bottom of the tank, which increased in SF when in visual contact with a predator as well as 
249 in their respective RF. We can confirm that zebrafish are capable of interpreting imminent

250 predation risk simply by viewing the behavior of their conspecifics when a predator is present,

251 which triggers an anti-predatory response. These results highlight the complexity of anti-predator

252 communication among zebrafish, given that defensive and stress responses can be triggered in

253 conspecifics that cannot see the predator directly and thus represent indirect responses to predation

254 risk. Communication based on visual cues aids zebrafish shoals in dealing with predators. Such

255 communication might take the form of a chain reaction, in which a single fish detects a predator

256 and responds to this threat with the defensive behavior consequently propagating throughout the

257 entire shoal. Likewise, it may even be that zebrafish displaying anti-predator responses in one

258 shoal can induce similar responses in fish in another shoal, given that copying is a commonly

259 observed behavior in shoal fish, for instance when the leader of a shoal "commands" the group

260 during foraging navigation (Reebs, 2000). Whether similar patterns occur for defensive reactions

261 deserves future investigation.

262

That zebrafish in visual contact with a predator have higher levels of whole-body cortisol

263

264

265

266

267

268

269

270

271 was previously reported by Barcellos et al. (2007), but surprisingly, zebrafish within visual contact of a non-predator fish presented a similar increase in cortisol levels. However, the cortisol response within this group (SF non-predator treatment) was of smaller magnitude than in zebrafish viewing a predator. Nonetheless, RF viewing these conspecifics had no elevated cortisol. Cortisol levels in SF exposed to non-predator fish were also elevated, but these fish exhibited similar behavior (time in the bottom section of the tank). Thus, since RF received only visual cues, this behavior failed to elicit a cortisol response. In the non-predator treatment, both SF and RF spent more time near the tank bottom compared to fish in the control group (absence of a stimulus fish), but this response was not as significant as that among fish in the predator-exposure treatment. 

behavioral and stress response in fish in the non-predator treatment may be due to limited predator; recognition by fish of a real predation risk may depend on other cues, such as scent

278 (Korpi \& Wisenden, 2001) or mechanical (Hegab \& Wei, 2014) cues. Visual recognition of a predator by prey fish is based on movement patterns characteristic of the predator (Barcellos et al., 2007); because our non-predator goldfish was very active, zebrafish may have initially mistaken it for a predator. Fish in our experiment were given only visual stimulus, and thus the absence of combinations of different cues may result in 'misinterpretation' of a harmless stimulus, leading to the observed increases in cortisol levels in SF in the non-predator treatment. Zebrafish that viewed conspecifics that in turn viewed a non-predator did not alter their behavior, and no cortisol response was triggered, supporting the 'misinterpretation' hypothesis. Our findings also highlight the need for care in relation to visual cues when using zebrafish in experiments. Other visual stimuli (e.g., presence of humans, other fish species, or even other types of animals) may be interpreted as potential threats by the fish, affecting their behavioral and hormonal patterns and consequently influencing experimental results. communication among conspecifics in response to predation risk in aquatic environments.

\section{References}


294 Allen PM. 1975. Darwinian evolution and a predator-prey ecology. Bull. Math. Biol. 37: 389-405.

295 DOI: 10.1007/BF02459521

296

297

298

299

300

301

302

303

304

305

306

307

308

309

310

311

312

313

314

315

316

317

318

319

320

321

Abril-de-Abreu R, Cruz J, Oliveira RF. 2015. Social eavesdropping in zebrafish: tuning of attention to social interactions. Sci. Rep. 5: 12678. DOI: 10.1038/srep12678

Archard GA, Earley RL, Hanninen AF, Braithwaite VA. 2012. Correlated behavior and stress physiology in fish exposed to different levels of predation pressure. Func. Ecol. 26: 637-645. DOI: 10.1111/j.1365-2435.2012.01968.x

Barcellos LJG, Ritter F, Kreutz LC, Quevedo RM, da Silva LB, Bedin AC, Finco J, Cericato L. 2007. Whole-body cortisol increases after direct and visual contact with the predator in zebrafish, Danio rerio. Aquaculture 272: 774-778. DOI: 10.1016/j.aquaculture.2007.09.002

Barcellos LJG, Volpato GL, Barreto RE, Coldebella I, Ferreira D. 2011. Chemical communication of handling stress in fish. Physiol. Behav. 103: 372-375. DOI: 10.1016/j.physbeh.2011.03.009

Barcellos LJG, Koakoski G, da Rosa JGS, Ferreira D, Barreto RE, Giaquinto PC, Volpato GL. 2014. Chemical communication of predation risk in zebrafish does not depend on cortisol increase. Sci. Rep. 4: 5076. DOI: 10.1038/srep05076

Barreto RE, Miyai CA, Sanches FHC, Giaquinto PC, Delcidio HC, Volpato GL. 2013. Blood cues induced antipredator behavior in Nile tilapia conspecifics. PloS One 8: e54642. DOI: 10.1371/journal.pone.0054642

Barreto RE, Luchiari AC, Marcondes AL. 2003. Ventilatory frequency indicates visual recognition of an allopatric predator in naive Nile tilapia. Behav. Proc. 60: 235-239. DOI: 10.1016/S03766357(02)00127-4

Barreto RE, Barbosa-Junior A, Urbinati EC, Hofmann A. 2014. Cortisol influences the antipredator behavior induced by chemical alarm cues in the frillfin goby. Horm. Behav. 65: 394400. DOI: 10.1016/j.yhbeh.2014.03.007

Barton BA.2002. Stress in fishes: a diversity of responses with particular references to changes in circulating corticosteroids. Integr. Comp. Biol. 42: 517-525. DOI: 10.1093/icb/42.3.517

Chivers DP, Smith RJF. 1998. Chemical alarm signaling in aquatic predator-prey systems: a review and prospectus. Ecoscience 5: 338-352. DOI: 10.1080/11956860.1998.11682471 
322 Clinchy M, Charlier TD, Newman AE, Schmidt KL, Boonstra R, Soma KK. 2011. Multiple 323 measures elucidate glucocorticoid responses to environmental variation in predation threat. 324 Oecologia 166 607-614. DOI: 10.1007/s00442-011-1915-2

325 Cooper WE, Lopez P, Martin J, Perez-Mellado V. 2012. Latency to flee from an immobile 326 predator: effects of predation risk and cost of immobility for the prey. Behav. Ecol. 23: 790-797. 327 DOI: $10.1093 /$ beheco/ars032

328

329

330

331

332

333

334

335

336

337

338

339

340

341

342

343

344 345

346

347

348

349

350

351

352

353

Cresswell W. 2010. Empirical studies of predator and prey behavior. Encyclopedia of Animal Behavior 633-638. DOI: 10.1016/B978-0-12-809633-8.01010-4

Delicio HC, Barreto RE. 2008. Time-place learning in food restricted Nile tilapia. Behav. Proc. 77: 126-130. DOI:10.1016/j.beproc.2007.06.005

Doutrelant C, McGregor PK. 2000. Eavesdropping and mate choice in female fighting fish. Behavior 137: 1655-1669. DOI: 10.1163/156853900502763

Dunlop-Hayden KL, Rehage JR. 2011. Antipredator behavior and cue recognition by multiple everglades prey to a novel cichlid predator. Behavior 148: 795-823.

Gerlai R, CsányiV. 1990. Genotype-environment interaction and the correlation structure of behavioral elements in paradise fish (Macropodus opercularis). Physiol. Behav. 47: 343-356. DOI: 10.1016/0031-9384(90)90153-U

Gerlai R, Lahav M, Guo S, Rosenthal A. 2000. Drinks like a fish: zebra fish (Danio rerio) as a behavior genetic model to study alcohol effects. Pharmacol. Biochem. Behav. 67: 773-782. DOI: 10.1016/S0091-3057(00)00422-6

Gerlai R. 2003. Zebra fish: an uncharted behavior genetic model. Behav. Gen. 33: 461-468. DOI: 10.1023/A:1025762314250

Gebauer DL, Pagnussat N, Piato AL, Schaefer IC, Bonan CD, Lara DR. 2011. Effects of anxiolytics in zebrafish: similarities and differences between benzodiazepines, buspirone and ethanol. Pharmacol. Biochem. Behav. 99: 480-486. DOI: 10.1016/j.pbb.2011.04.021.

Jordão LC. \& Volpato GL. 2000. Chemical transfer of warning information in non-injured fish. Behavior 137: 681-690. DOI: 10.1163/156853900502286

Hegab IM, Wei W. 2014. Neuroendocrine changes upon exposure to predator odors. Physiol. Behav. 131: 149-155. DOI: 10.1016/j.physbeh.2014.04.041 
354 Kalluef AV, Stewart AM, Gerlai R. 2014. Zebrafish as an emerging model for studying complex 355 brain disorders. Cell Press 35: 63-75. DOI: 10.1016/j.tips.2013.12.002.

356 Korpi NL, Wisenden BD. 2001. Learned recognition of novel predator odour by zebra danio, 357 Danio rerio, following time-shifted presentation of alarm cue and predator odour. Environ. Biol. 358 Fish. 61: 205-211. DOI:10.1023/A:1011091426030

359

360

361

362

363

364

365

366

367

368

369

370

371

372

373

374

375

376

377

378

379

380

381

382

383

384

385

Magrath RD, Haff TM, Fallow PM, Radford AN. 2015. Eavesdropping on heterospecificic alarm calls: from mechanisms to consequences. Biol. Rev. 90: 560-586. DOI: 10.1111/brv.12122

Martin JM, Saaristo M, Bertram MG, Lewis PJ, Coggan TL, Clarke BO, Wong BBM. 2017. The psychoactive pollutant fluoxetine compromises antipredator behavior in fish. Environ. Pollut. 222: 592-599. DOI: 10.1016/j.envpol.2016.10.010

Meshalkina DA, Kizlyuk M, Kisel E, Collier AD, Echevarria DJ, Abreu MS, Barcellos LJG, Song C, Warnick JE, Kyzar EJ, Kalluef AV. 2017. Zebrafish models of autism spectrum disorders. Exp. Neurol. DOI: 10.1016/j.expneurol.2017.02.004

Miyai CA, Carretero Sanches FH, Costa TM, Colpo KD, Volpato GL, Barreto RE. 2011. The correlation between subordinate fish eye colour and received attacks: a negative social feedback mechanism for the reduction of aggression during the formation of dominance hierarchies. J. Zool. 114: 335-339. DOI: 10.1016/j.zool.2011.07.001

Morishita VR, Barreto RE. 2011. Black sea urchins evaluate predation risk using chemical signals from a predator and injured con- and heterospecific prey. Mar. Ecol. Progr. Series 435: 173-181. DOI: $10.3354 /$ meps09253

Mullan R, Glass DH, McCartney M. 2015.Species diversity and predation strategies in a multiple species predator-prey model. Commun. Nonlinear Sci. Numer. Simul. 25: 118-135. DOI: 10.1016/j.cnsns.2015.01.011

Maximino C, Brito TM, Dias CAGM, Gouveia Jr A, Morato S. 2010. Scototaxis as anxiety-like behavior in fish. Nat. Prot. 5: 209-216. DOI: 10.1038/nprot.2009.225

Miller N, Gerlai R.2007. Quantification of shoaling behavior in zebrafish (Danio rerio). Behav. Brain Res. 184: 157-166. DOI: 10.1016/j.bbr.2007.07.007

O'Connor CM, Reddon AR, Odetunde A, Jindal S, Balshine S. 2015. Social cichlid fish change behavior in response to a visual predator stimulus, but not the odour of damaged conspecifics. Behav. Processes 121: 21-29. DOI: 10.1016/j.beproc.2015.10.002 
386 Oliveira TA, Koakoski G, da Motta AC, Piato ALS, Barreto RE, Volpato GL, Barcellos LJG. 387 2014. Death-associated odors induce stress in zebrafish. Horm. Behav. 65: 340-344. DOI: 388 10.1016/j.yhbeh.2014.02.009

389 Oliveira TA, Koakoski G, Kreutz LC, Ferreira D, Santos da Rosa JG, de Abre MS, Giacomini 390 ACV, Oliveira RP, Fagundes M, Piato AL, Barreto RE, Barcellos LJG. 2013. Alcohol impairs 391 predation risk response and communication in zebrafish. PloS One 8: e75780. DOI: 392 10.1371/journal.pone.0075780

393 Quadros VA, Silveira A, Giuliani GS, Didonet F, Silveira AS, Nunes ME, Silva TO, Loro VL, 394 Rosemberg DB. 2016. Strain- and context-dependent behavioral responses of acute alarm subtance 395 exposure in zebrafish. Behav. Proc. 122: 1-11. DOI: 10.1016/j.beproc.2015.10.014

396 Reebs SG. 2000. Can a minority of informed leaders determine the foraging movements of a fish 397 shoal? Anim. Behav. 59: 403-409. DOI: 10.1006/anbe.1999

398

399

400

401

402

403

404

405
Sink TD, Lochmann RT, Fecteau KA. 2007. Validation, use, and disadvantages of enzyme-linked immunosorbent assay kits for detection of cortisol in channel catfish, largemouth bass, red pacu and golden shiners. Fish Physiol. Biochem. 75: 165-171. DOI: 10.1007/s10695-007-9150-9

Speedie N, Gerlai R.2008. Alarm substance induced behavioral responses in zebrafish (Danio rerio). Behav. Brain Res. 188: 168-177. DOI: 10.1016/j.bbr.2007.10.031

Wisendem BD, Vollbrechet KA, Brown JL. 2004. Is there a fish alarm cue? Affirming evidence from a wild study. Anim. Behav. 67: 59-67. DOI: 10.1016/j.anbehav.2003.02.010 